\title{
Conocimientos y percepciones de niñas, niños y adolescentes sobre la sexualidad
}

\author{
Notions and perceptions of girls, boys and adolescents about sexuality
}

\section{Noções e percepções de meninas, meninos e adolescentes sobre sexualidade}

\author{
Daniela Roldán Restrepo ${ }^{1}$, Andrea Arenas Duque², Luz Janeth Forero Martínez³, Danny Rivera ${ }^{4}$, Juan Carlos Rivillas ${ }^{5}$ \\ Socióloga. Asociación Profamilia. Bogotá, Colombia. daniela.roldan@profamilia.org.co. ORCID: https://orcid.org/0000-0001-8577-8978 \\ 2 Economista y negociadora internacional, magíster en Salud Pública. Asociación Profamilia. Bogotá, Colombia. andrea.arenas@ \\ profamilia.org.co. ORCID: https://orcid.org/0000-0002-1742-6230 \\ 3 Médica cirujana, doctora en Sociología Jurídica e Instituciones Políticas. Asociación Profamilia. Bogotá, Colombia. Iuzjaneth.forero@ \\ profamilia.org.co. ORCID: https://orcid.org/0000-0002-9379-836X \\ $4 \quad$ Estadístico. Asociación Profamilia. Bogotá, Colombia. danny.rivera@profamilia.org.co. oncid: https://orcid.org/0000-0001-7736-2411 \\ 5 Administrador en Servicios de Salud, magíster en Economía y Política de la Salud. Asociación Profamilia. Bogotá, Colombia. juan. \\ rivillas@profamilia.org.co. ORCID: https://orcid.org/0000-0002-0961-3079
}

Recibido: 23/07/2019. Aprobado: 08/06/2020 . Publicado: 30/10/2020

Roldán-Restrepo D, Arenas Duque A, Forero Martínez LJ, Rivera D, Rivillas JC. Conocimientos y percepciones de niñas, niños y adolescentes sobre la sexualidad. Rev. Fac. Nac. Salud Pública. 2020;39(2):e339044. Dor: https://doi.org/10.17533/udea.rfnsp. e339044

\section{Resumen}

Objetivo: Explorar los conocimientos y las percepciones de algunas niñas, niños y adolescentes respecto a la sexualidad, el ejercicio de los derechos sexuales y reproductivos, y la educación sexual. Metodología: se realizó un estudio cualitativo, a partir de 25 entrevistas semiestructuradas a personas de entre 9 y 14 años, en 8 municipios priorizados de Colombia. Resultados: En todos los municipios se encontró un desconocimiento generalizado de los temas abordados, así como significados reducidos y percepciones en extremo negativas sobre la sexualidad, la educación sexual, los derechos sexuales y reproductivos, y la salud sexual, a lo que se suman fuertes estereotipos de género. Conclusiones: Los conocimientos y las connotaciones negativas asignadas a los conceptos por los que se preguntó dan cuenta de la limitada y precaria educación sexual que han recibido niñas, niños $\mathrm{y}$ adolescentes de estos municipios, lo que significa que aunque en el país existe un marco legal propicio y unas metas relacionadas con el abordaje integral de la sexualidad desde la primera infancia, estos no se están cumpliendo.

Palabras clave: Sexualidad, infancia, educación sexual, derechos sexuales y reproductivos, salud sexual. 


\begin{abstract}
Objective: To explore children knowledge and perceptions about sexuality, exercise of sexual and reproductive rights and sex education. Methods: A qualitative study was conducted through 25 semi-structured interviews with girls, boys and adolescents from 9 to 14 years old, in eight prioritized municipalities in Colombia. Results: In all the municipalities there was a widespread ignorance about the issues addressed, as well as reduced meanings and extremely negative perceptions about sexuality, sex education and sexual and reproductive rights and health; additionally were found strong
\end{abstract}

gender stereotypes. Conclusions: perceptions and negative connotations assigned to the concepts that were inquired, accounted the limited and precarious sex education that girls and boys from these municipalities have received, which mean that, despite that in the country there is a favorable legal framework and goals related to the comprehensive approach of sexuality from early childhood, these are not being met.

Keywords: Sexuality, Childhood, Perceptions, Sexual Education, Sexual and Reproductive Rights

\section{Resumo}

Objetivo: Explorar o conhecimiento e as percepções de meninas e meninos sobre a sexualidade, o exercício dos direitos sexuais e reprodutivos e a educação sexual. Métodos: Estudo qualitativo realizado por meio de entrevistas semiestruturadas com 25 meninas, meninos e adolescentes entre os nove e os 14 anos, em oito municípios priorizados da Colômbia. Resultados: Em todos os municípios foi encontrado um desconhecimiento generalisado sobre as questões abordadas, bem como significados reduzidos e percepções extremadamente negativas sobre a sexualidade, a educação sexual, os direitos esxuais e reprodutivos e a saúde sexual, e também fortos estereótipos de gênero. Conclusões: Os conhecimentos e as conotações negativas atribuídas aos conceitos o que foram preguntados, dão conta da educação sexual limitada e precária recebida por meninos, meninas e adolescentes desses municipios, o que significa, que a pesar de que o país tem um marco legal favorável e metas relacionadas com o abordagem integral da sexualidade desde a prmeira infancia, estes não estão sendo atendidos.

Palavras-chave: Sexualidade, Infância, Percepções, Educação Sexual, Direitos Sexuais e Reprodutivos

\section{Introducción}

Niñas, niños y adolescentes son, en la actualidad, reconocidos como sujetos con voz y derechos. Algunos hitos de este reconocimiento los constituyen la "Declaración de Ginebra" (1924), la "Declaración de los Derechos del Niño" (1959) y la "Convención sobre los Derechos del Niño" (1989) [1], en los que, sin embargo, hay un vacío respecto a los derechos sexuales y reproductivos (DSR) de las niñas, los niños y las y los adolescentes [2].

En Colombia, el marco legal plantea que es necesario trabajar por la garantía de los DSR de las niñas, los niños y las y los adolescentes [3] y normatiza la incorporación de la educación sexual en todos los niveles escolares, desde la primera infancia [4]. No obstante, la implementación de los programas integrales para su educación sexual ha sido baja, así como limitado el involucramiento de las partes implicadas [5]. Según Vargas Trujillo, esto se debe al tabú frente a la sexualidad de las niñas, los niños y las y los adolescentes, basado, a su vez, en la comprensión generalizada de la sexualidad como genitalidad [6], comprensión que va en contravía de una definición amplia de la misma, como la propuesta por la Organización Mundial de la Salud: "aspecto central del ser humano, presente a lo largo de su vida, que abarca al sexo, las identidades y los papeles de género, el erotismo, el placer, la intimidad, la reproducción y la orientación sexual" [7].

De hecho, existe evidencia de que la educación para la sexualidad en los niveles iniciales se ha dejado de lado. La "Encuesta nacional de demografía y salud", de 2015 , muestra que la edad promedio a la que se accede por primera vez a información sobre sexualidad es de 15,5 años para los hombres y de 16,2 años para las mujeres $[8$, p. 550]. A estas asimetrías de género se suman otros determinantes sociales, que hacen que el acceso a la información sobre sexualidad sea aún más tardío, como residir en zonas rurales o ubicarse en el quintil más bajo de riqueza $[8,9]$. 
El tabú respecto a la sexualidad de las niñas, los niños y las y los adolescentes es tan fuerte, que incluso dentro del campo académico, en los estudios sobre la infancia, la sexualidad generalmente es eludida [10]. En los casos en los que es considerada, se la circunscribe a un marco proteccionista y a una visión de la misma como algo que es peligroso o de lo que debería protegérseles [10].

Por todo lo anterior, la Asociación Profamilia, ${ }^{*}$ con el apoyo de la embajada de Canadá, diseñó el Proyecto "Valiente: garantizando los derechos de las niñas a través del fortalecimiento de la educación integral para la sexualidad (2019-2023)" [11], dirigido a niñas, niños y adolescentes entre 9 y 14 años, de 8 municipios de Colombia: Aracataca, Bahía Solano, Caloto, Dibulla, El Carmen de Atrato, Miranda, Pivijay y Uribia. Estos municipios hacen parte de los departamentos de Colombia con mayores porcentajes de población pobre: Chocó $(61,1 \%)$, La Guajira (53,7 \%), Cauca (50,5 \%) y Magdalena (46,6 \%) [12], y presentan altas tasas de embarazo en adolescentes: mientras el porcentaje de mujeres entre 13 y 19 años que ya son madres es 8,8 \% para Bogotá, en La Guajira es 16,6 \%, en Magdalena 20,9 \%, en Cauca 21,1\% y en Chocó alcanza el $24 \%$ [8]. En este proyecto, la educación sexual de calidad es entendida como un medio para prevenir los resultados negativos de no contar con esta información, pero también como un proceso continuo de reflexión que permita conocerse y reconocerse a sí mismo y a los otros como sujetos de derechos sexuales y reproductivos, civiles y políticos.

El objetivo general de la investigación que se reporta en este artículo fue explorar los conocimientos y las percepciones de algunas niñas, niños y adolescentes respecto a la sexualidad, el ejercicio de los derechos sexuales y reproductivos, y la educación sexual.

\section{Metodología}

La investigación fue de tipo cualitativa y se basó en una perspectiva constructivista, según la cual la realidad y el conocimiento sobre la misma se construyen en y mediante relaciones interpersonales. Así pues, sin la pretensión de objetividad propia de otras perspectivas (como la positivista o empirista), se efectuaron 25 entrevistas semiestructuradas en profundidad, con niñas y adolescentes mujeres $(n=15)$ y niños y adolescentes varones $(n=10)$, quienes se seleccionaron mediante muestreo por conveniencia, teniendo como criterio que fueran susceptibles de hacer parte de la intervención de educación integral para la sexualidad a implementar por la Asociación Profamilia, esto es: 1) que estuvieran escolarizados; 2) que tuvieran entre 9 y 14 años de edad; y 3) que fueran habitantes de los 8 municipios seleccionados para el Proyecto: Aracataca y Pivijay (Magdalena), Bahía Solano y El Carmen de Atrato (Chocó), Caloto y Miranda (El Cauca) y Dibulla y Uribia (La Guajira).

Las entrevistas se realizaron entre octubre de 2018 y febrero de 2019, después de someter los instrumentos a una prueba piloto. Las niñas, los niños y las y los adolescentes entrevistados fueron contactados en sus actividades rutinarias de colegio. Las entrevistas se llevaron a cabo de manera individual, en un espacio privado.

Desde la perspectiva constructivista se cuestiona la posibilidad de acceder a información objetiva o pura. La producción de sentido se da en la interacción entre entrevistado y entrevistadora, y quienes son entrevistados son consideradas personas con agencia y con capacidad para rechazar premisas e incluso preguntas. Sin embargo, se tomaron las precauciones corrientes para controlar la inducción de respuestas en la realización de entrevistas, como: 1) que las personas no conozcan previamente las preguntas, 2) que se aclare que no existen preguntas "correctas" o "incorrectas", y 3 ) evitar el uso de reforzadores positivos o negativos (verbales y no verbales), como estimular o celebrar ciertas ideas, y rechazar otras.

Adicionalmente, para posibilitar la escucha activa y evitar sesgos de transcripción, no se tomaron notas durante las entrevistas, sino que estas fueron grabadas, anonimizadas y luego transcritas textualmente por una persona externa. Una vez transcritas, las entrevistas fueron procesadas con el software $\mathrm{NVivo}^{\circledR}$ (QSR International, Melbourne, versión 12.6.0).

El análisis se basó en la metodología de la teoría fundamentada, que permite construir códigos y categorías a partir de la observación de los datos recolectados. De acuerdo con el objetivo de la investigación y las preguntas realizadas, en un primer momento la información se clasificó temáticamente, para luego hacer una lectura detallada de los datos y construir códigos, como: nociones y definiciones de sexualidad, o necesidades en salud sexual y reproductiva. La densidad y la concentración de códigos dieron lugar a categorías como: percepciones sobre sexualidad; personas de confianza para hablar sobre sexualidad; estereotipos de género; actitud frente a las diversidades sexuales y de género; temores a la atención en privado por el personal de la salud y percepciones sobre el trato del personal de la salud.

* Fundada en 1965, Profamilia es una organización privada sin ánimo de lucro, que promueve el respeto y el ejercicio de los derechos sexuales y derechos reproductivos de toda la población colombiana. 
La investigación fue aprobada por el Comité de Ética de Investigaciones de Profamilia, mediante el Acta 61 del 17 de octubre de 2018, y se basó en los fundamentos científicos y éticos contemplados en las normas nacionales e internacionales para la protección de los derechos de participantes menores de edad, quienes manifestaron, de manera escrita y voluntaria, su deseo de participar. $^{\dagger}$

\section{Resultados}

\section{Nociones y percepciones de niñas, niños y adolescentes frente a la sexualidad, los derechos sexuales $y$ reproductivos, y la educación sexual}

Entrevistadora: ¿Qué se te ocurre cuando escuchas la palabra "sexualidad"?

Niña: No la he escuchado.

Entrevistadora: Y ya que yo te la estoy diciendo, cuando yo te digo "sexualidad", a ti, eso, ¿a qué te suena?

Niña: Como a hacer cosas malas (Niña, 10 años, Pivijay).

Para las niñas, los niños y las y los adolescentes que participaron de las entrevistas, la sexualidad es un tema sobre el que prefieren no hablar. Durante las conversaciones, esto se observó tanto en su expresión corporal - de las evasivas y de las respuestas tajantes - como de manera explícita al momento de justificar su preferencia por pasar a otro tema de conversación o, una vez finalizada la entrevista, cuando se les preguntó por cómo se habían sentido durante la misma.

La incomodidad fue un primer indicio del poco dominio de las niñas, los niños y las y los adolescentes sobre el tema y del significado acotado y negativo que atribuyen a la sexualidad y a los asuntos relacionados con la palabra "sexual", como educación sexual o derechos sexuales. En la Figura 1 se muestra que, en la mayoría de los casos, las persona entrevistadas definieron la sexualidad de manera limitada, relacionando el concepto con el contacto y la genitalidad. Así, para la mayoría, sexualidad es hacer el amor o tener relaciones sexuales, no necesariamente penetrativas, pues el contacto también fue concebido en varios casos como un acto sexual. De especial mención es el hecho de que las niñas y adolescentes mujeres, en mayor proporción que los niños y adolescentes varones, definieran la sexualidad como abuso o violencia sexual.

En las entrevistas, la sexualidad no solo estuvo definida de manera limitada, sino también negativa. La definición de la sexualidad como abuso fue quizá la forma más extrema en la que esto se manifestó, pero esta fue una percepción compartida por niñas, niños y adolescentes, quienes en algunos casos definieron la sexualidad como "hacer cosas malas" o "hacer groserías". En contraste, fueron pocos los casos en los que se encontraron definiciones de la sexualidad ligadas a la identidad o la autonomía, y en ningún caso se hizo alusión a la libertad o el goce.

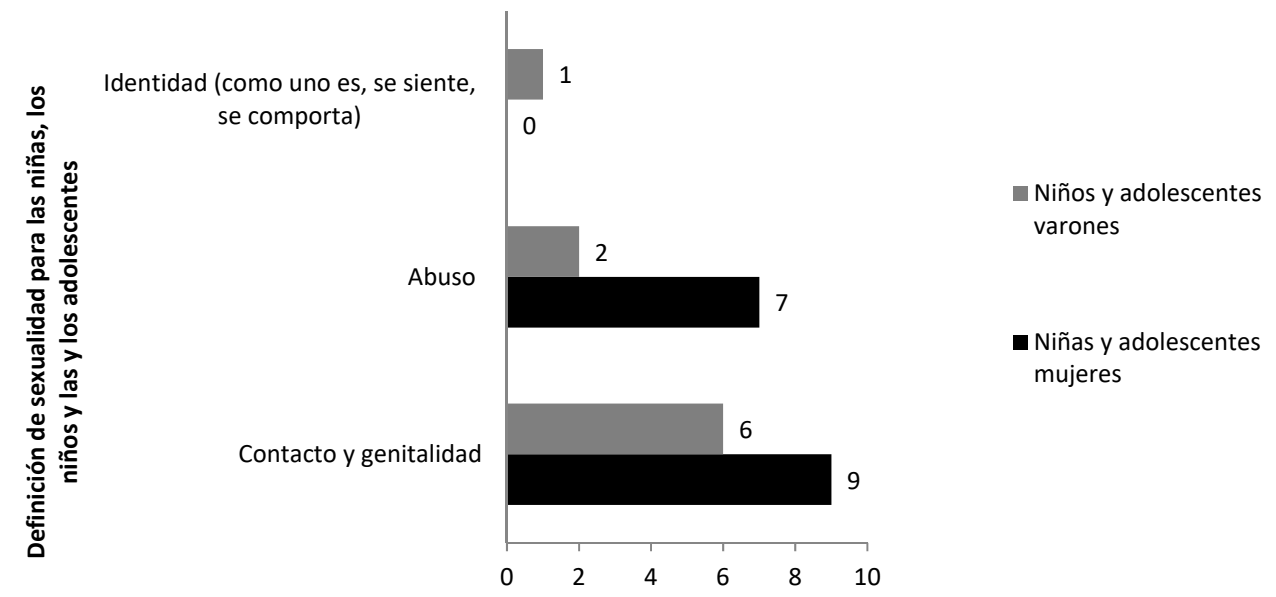

Figura 1. Categorías a las que más referencia hicieron las personas entrevistadas según sexo.

En la "Pauta 17" de las "Pautas éticas internacionales para la investigación relacionada con la salud con seres humanos", se establecen los lineamientos éticos a tener en cuenta cuando la investigación implica la participación de niñas, niños y adolescentes, casos en los que se requiere su asentimiento informado, además del consentimiento de sus madres, padres o responsables legales. La obtención de este solo se dispensa cuando "el conocimiento del tema de la investigación por parte de los padres pueda colocar a los niños o los adolescentes en riesgo de que sus padres los interroguen, los intimiden o les causen daño" [13, p. 76]. 
En lo que respecta a los DSR, el desconocimiento fue el común denominador, más que las nociones vagas o limitadas. Por esta razón, durante las entrevistas fue necesario construir, con las y los participantes, el concepto de derecho, al que se agregaron luego las palabras "sexuales" y "reproductivos". Esto resultó en que se obtuvieran definiciones literales o que en algunos casos la palabra "sexual" tornara negativo el concepto de derecho que inicialmente había sido definido en positivo.

En algunos casos, la edad fue señalada como un límite para considerarse a sí mismos y mismas titulares de DSR. Esto puede observarse en el siguiente fragmento de una entrevista en la que, además, se evidencia la percepción negativa sobre la sexualidad, que fue mayoritaria en las niñas, los niños y las y los adolescentes:

Entrevistadora: $\mathrm{Y}$ entonces, si eso que tú me dijiste es lo que tú piensas que son los derechos, ¿qué crees que son los derechos sexuales y reproductivos?

Niño: Mi mamá dice que si yo soy chiquito, que si tengo novia, no me puedo ir a dar besitos con ella, ni hacer nada de esas cosas malas; pero si soy grande, yo me mando por sí mismo y puedo hacer lo que quiera.

Entrevistadora: Entonces, ¿tendrías derechos sexuales y reproductivos?

Niño: Si soy grande... (Niño, 10 años, El Carmen de Atrato).

Igualmente, las niñas, los niños y las y los adolescentes mostraron un profundo desconocimiento cuando se les preguntó por la educación sexual. Según ellas y ellos, nunca ha sido recibida una información amplia y estructurada sobre el tema. Solo en algunos casos, las personas entrevistadas mayores de 12 años se refirieron a materias en las que el tema había sido abordado. Estas materias o cursos fueron, en su mayoría, ciencias naturales y ética y valores. Cuando se profundizó sobre esto, fue notorio que se trataba de procesos formativos poco sistemáticos.

El énfasis de los mensajes que las niñas, los niños y las y los adolescentes reciben sobre sexualidad está puesto en consideraciones morales y en aspectos biológicos de la sexualidad, que se centran en aplazar el inicio de la actividad sexual y en prevenir los riesgos asociados. A las niñas se las educa en la culpa, y en el miedo a los hombres, a través de mensajes en los que estos son presentados como algo de lo que ellas deben cuidarse. Como se observa en los siguientes fragmentos, cuando las niñas dieron cuenta de aquello que han oído en relación con la educación sexual, sus respuestas estuvieron cargadas de referencias al peligro de violencia sexual y a la responsabilidad que tienen de cuidarse de él:

Entrevistadora: ¿Alguien más te ha hablado de educación sexual?
Niña: Mi mamá.

Entrevistadora: ¿Tú mamá? ¿Y qué te dice?

Niña: Que las niñas nunca se deben dejar tocar de los hombres... (Niña, 10 años, Aracataca).

Entrevistadora: Dime lo que te acuerdes que te hayan dicho y que hayas aprendido, que tú hayas aprendido.

Niña: Que debo cuidar mi cuerpo.

Entrevistadora: ¿Debes cuidarlo de qué?

Niña: De los hombres, por ejemplo, de los violadores (Niña, 12 años, Dibulla).

En contraste y según sus discursos, a los niños y adolescentes varones se los alerta predominantemente sobre las infecciones de transmisión sexual (ITS) y el embarazo en la adolescencia. En ningún caso, las niñas, los niños y las y los adolescentes hicieron referencia a mensajes relacionados con el enfoque de derechos de la educación sexual, ausencia que pudo notarse en su desconocimiento de los DSR. De allí que, en su mayoría, definieran la educación sexual en los términos en los que se les ha hablado de la misma, sin trascender el enfoque de riesgo.

El correlato de este énfasis se encontró en al menos tres elementos que emergieron constantemente en los discursos de las niñas, los niños y las y los adolescentes al hablar de la educación sexual y de sus expectativas respecto a esta: 1) la percepción de la sexualidad como algo malo o sucio; 2) el afán por saber cómo comportarse "bien", que fue, en muchos casos, homologado a cómo contener el deseo sexual o abstenerse de tener relaciones sexuales, y 3) la preocupación por las ITs o el embarazo a temprana edad, a la que se sumó, en el caso de las niñas, la preocupación por aprender a cómo cuidarse de los hombres y del abuso, y a cómo denunciarlo.

La información sobre la sexualidad que las personas entrevistadas han recibido responde a acciones educativas no intencionales, es decir, de "carácter intuitivo, asistemático y poco estructurado" [8]. Más que la escuela y las profesoras, las familias (específicamente las madres) fueron la institución que las niñas, los niños y las y los adolescentes señalaron como principal fuente de información sobre sexualidad. En contraste, pares y amigas y amigos fueron vistos como personas con las que es mejor no hablar del tema, porque se percibe que estos no pueden preservar la confidencialidad, atributo consustancial a la sexualidad para las niñas, los niños y las y los adolescentes.

La televisión emergió como otro de los medios principales a través de los cuales las personas entrevistadas reciben información en sexualidad. Por ejemplo, al indagar por qué una niña había definido la sexualidad como violación, se encontró que se basaba en una escena de abuso sexual vista en una telenovela. 


\section{"A nosotras nos gusta el rosado, a ellos el azul". Roles y estereotipos de género}

Los estereotipos de género son la construcción social y cultural respecto a lo que implica ser "hombres" y ser "mujeres" en razón de las diferencias físicas, biológicas, sexuales y sociales [14]. La definición amplia de estos estereotipos incluye una diferenciación analítica entre: 1) estereotipos de sexo, donde los hombres y las mujeres poseen características físicas diferenciadas; 2) estereotipos sexuales, que determinan las formas aceptables de interacción sexual entre hombres y mujeres, y 3) estereotipos sobre los roles sexuales, que prescriben los comportamientos esperados para hombres y mujeres [14].

En muchos casos, sin que las preguntas se orientaran a ello, los estereotipos de género emergieron constantemente. Estos se exploraron al interrogar por la manera en la que las niñas, los niños y las y los adolescentes se autoidentifican y por las cosas que califican como propias y posibles para su sexo/género. La referencia al tipo de juegos y a los opuestos fue constante en sus discursos para llegar a estos significados.

La actitud y la expresión corporal de las niñas, los niños y las y los adolescentes ante las preguntas relacionadas con esta temática fue más relajada. Incluso, cuando no sabían qué responder, permitieron "pensar en voz alta" para encontrar sus respuestas, contar historias, hacer bromas y emitir juicios de valor.

Los estereotipos que más se relacionaron con la diferencia sexual se hallaron en las niñas, pero hicieron parte de las primeras nociones por medio de las cuales las niñas, los niños y las y los adolescentes en general se definieron a sí mismos. Aquí, las respuestas estuvieron guiadas por una lógica binaria de lo sexual e incluyeron referencias a características físicas, como la longitud del cabello, el uso de aretes y los genitales. Estereotipos de sexo, sexuales y sobre roles se encuentran imbricados, como en el siguiente fragmento, en el que la entrevistada se refiere a lo que es "normal" para una niña.

Entrevistadora: Te ves como una niña, ¿y qué significa eso para ti?

Niña: Ser femenina, tener lo de una niña normal, hacer lo de una niña normal.

Entrevistadora: ¿Y qué es lo que tiene y hace una niña normal?

Niña: La niña normal tiene... tiene sus partes, que no lo tienen los niños igual. Tenemos una personalidad diferente; por ejemplo, a nosotras nos gusta el rosado, a ellos el azul, y así.

Entrevistadora: ¿Y qué más les gusta a las niñas?

Niña: Nos gusta bailar, a ellos también, pero no tanto; en cambio, a todas nos gusta bailar y nos gusta jugar con muñecas; ellos juegan carritos, nosotras con bar- bies, y ellos son muy bruscos; así, cuando uno está jugando, uno es más delicada (Niña, 11 años, Uribia).

En la mayoría de las entrevistas, se relacionó el ser mujer con la delicadeza, la compasión, la alegría, la empatía, la debilidad física y el miedo, mientras que el varón fue asociado con la agresividad, el desorden, la amargura y la fuerza física. El binarismo sexual, que se encuentra en el modo en el que niñas, niños y adolescentes se definen como de uno y otro sexo, termina por dictar cuáles son las formas "normales" o "correctas" de ser y de comportarse. En estricta relación con ello, la heterosexualidad emergió como una norma que las niñas, los niños y las y los adolescentes se esmeran en cumplir.

Ahora bien, ya que, en la mayoría de los casos, la edad fue vista como un límite para sentir atracción sexual o relacionarse sexualmente con otras personas, la forma en la que las niñas, los niños y las y los adolescentes se muestran como heterosexuales es a través del rechazo y la discriminación a las diversidades sexuales y de género, por ejemplo, mediante el uso de los términos "gay", "marica" y "homosexual" como insulto. La explicación que dio una niña para no querer jugar con otra es ilustrativa de este comportamiento: "no me gusta jugar con ella porque parece un macho”.

La manera en la que las personas entrevistadas reivindican el binarismo sexual y la heteronormatividad se encuentra en los roles fuertemente estereotipados como opuestos complementarios - que mencionaron al responder a la pregunta por las cosas que son, a su juicio, de niñas o de niños:

Entrevistadora: ¿Qué cosas pueden o deberían hacer los niños?

Niño: Como ayudarle a trabajar a la mujer, o sea, uno da la plata para las mujeres y las mujeres van comprando.

Entrevistadora: ¿Y qué cosas crees que pueden o deberían hacer las niñas?

Niño: Ayudarle al hombre a veces.

Entrevistadora: ¿Cómo?

Niño: ¿Cómo le explico? Como a ayudarle a hacer el oficio en la casa $y$, mientras tanto, el hombre va trabajando ayudando a la mujer (Niño, 10 años, Bahía Solano).

Tan presentes están los estereotipos de género en el imaginario de las niñas, los niños y las y los adolescentes, que cuando se les preguntó si les gustaría hacer alguna de las cosas que señalaron como las propias del sexo opuesto, hubo pocas respuestas afirmativas. Las justificaciones para que esto fuera así no se pidieron explícitamente, pero fueron dadas, tal vez en un afán por reafirmar su feminidad/masculinidad. La lógica tautológica que se observa en el siguiente fragmento es ilustrativa del binarismo sexual o dicotomía hombre-mujer, 
sostenido, en términos generales, por las niñas y los niños entrevistados:

No me gustan esas cosas, porque son solo de mujeres, y lo de las mujeres son de parte de las mujeres y lo de hombres son de los hombres, más no. Los hombres no deben hacer las cosas que hacen las mujeres, sino las cosas que hacen los hombres, y las mujeres no pueden hacer lo que hacen los hombres, porque ellas tienen que hacer lo que hacen las mujeres (Niño, 10 años, Uribia).

El recurso a separaciones tajantes entre lo que es ser mujer y lo que es ser hombre, o lo que es de niñas y lo que es de niños, da cuenta de las limitaciones a las que unas y otros se enfrentan al momento de acceder a modelos de identificación más amplios o diversos y de vivir su corporalidad con autonomía. Esto es relevante, al observar cómo la identificación en términos dicotómicos lleva a que las niñas y los niños se vean a sí mismos como posibles víctimas y victimarios de la violencia de género. Así, entre las muchas respuestas que dieron para hallar el significado de ser una niña o un niño, también se encontraron referencias a estas posiciones:

Entrevistadora: A diferencia de ser un niño, por ejemplo, ¿qué significa ser niña?

Niña: Pues, yo digo que, pues, nosotras las mujeres somos las que más sentimos dolor, pues... digo yo, que los hombres no... yo... pues no se me viene nada más a la mente.

Entrevistadora: ¿Y dolor, como por qué, o en qué circunstancias?

Niña: Porque, por ejemplo... ay no entiendo... no sé cómo explicar.

Entrevistadora: No, tranquila. Tenemos todo el tiempo.

Niña: Porque a las mujeres las pueden maltratar y a los niños no, a ellos no (Niña, 13 años, El Carmen de Atrato).

La relación de niñas, niños y adolescentes con la salud sexual y reproductiva

En términos generales, para las niñas, los niños y las y los adolescentes entrevistados no existe necesidad de servicios SSR amigables, porque perciben que la atención en salud que reciben es suficiente y no sienten preferencia por recibir una atención en privado. Sin embargo, al indagar por las razones de estas percepciones y preferencias, se encontraron elementos a tener en cuenta, si se quiere contar con servicios de SSR amigables con esta población, para que sean conocidos y demandados por ella. El desconocimiento de los DSR, el temor a la privacidad del consultorio y el trato distante o en extremo cuidadoso son algunos de estos elementos.

Pese al temor a los embarazos, a las ITS y al abuso sexual que manifestaron durante las conversaciones, el desconocimiento que las niñas, los niños y las y los adolescentes tienen sobre los DSR influye de manera negativa en su salud sexual y reproductiva, porque no hay consciencia de su relación con los derechos. Así, las formas en las que afrontan sus temores son limitadas, muchas veces, porque no saben que son temas sobre los que pueden hablar y para los que existen personas capacitadas a las cuales acudir. Incluso, en algunos casos fue difícil hablar de las partes privadas de su cuerpo, porque consideraron que las palabras correctas para nombrarlas eran vulgares.

Independientemente de que existan personas capacitadas para responder a sus preguntas o necesidades en SSR, existe un temor que puede alejar a las niñas, los niños y las y los adolescentes de acudir a estas personas: el temor a la privacidad del consultorio. Esta fue otra de las razones por las cuales las personas entrevistadas no usan ni perciben como necesarios estos servicios. Durante las entrevistas, el temor se expresó tanto en términos de estar expuestas o expuestos a que pasen "cosas malas", como en términos de no saber qué hacer ante preguntas puntuales que pudieran hacerles, como: número de la tarjeta de identidad, dirección o teléfono. Algunos temores se relacionaron con las cosas que podrían pasarles durante el trayecto si fueran solas o solos a los centros de salud.

Otro elemento a tener en cuenta en lo que respecta a los servicios de SSR para niñas, niños y adolescentes es la forma en la que perciben cómo se les trata. De acuerdo con las conversaciones sostenidas, es necesario lograr un balance entre el trato cálido, de modo que no se sientan reprendidos por la distancia que les supone para la visión del personal médico (personas adultas, desconocidas y con conocimiento especializado) y el trato respetuoso de sus capacidades, de modo que no se les infantilice.

El respeto por sus decisiones y la necesidad de poder elegir entre médicas o médicos al momento de la atención cuando requieren un servicio de SSR, fueron algunas necesidades identificadas asociadas al trato. Las niñas y las adolescentes mujeres, en especial, manifestaron preferencia por ser atendidas por mujeres. Reconocer estas necesidades y darles lugar, puede hacer la diferencia entre un servicio amigable y uno que perciban como violento.

\section{Discusión}

Aunque en la región de América Latina son pocas las investigaciones cualitativas llevadas a cabo sobre la sexualidad y la educación sexual de niñas y niños preadolescentes (en contraste con la dedicada a quienes se encuentran en la adolescencia tardía), hay evidencia de que, desde edades tempranas, las personas ya tienen significados sobre la sexualidad, construidos a partir de 
los discursos hegemónicos de sus referentes cercanos $[15,16]$. Como se observó en las 25 entrevistas en profundidad realizadas, estos discursos hegemónicos suelen fundarse en una comprensión limitada de la sexualidad como genitalidad o acto sexual, y en la atribución de connotaciones negativas, como algo malo o sucio, y reservado a la adultez.

Estos discursos producen un tipo específico de educación sobre la sexualidad para niñas, niños y adolescentes: una educación basada en enfoques restrictivos (peligro o riesgo), que pone el énfasis en el aspecto físico de la sexualidad y que deja de lado sus aspectos cognitivos, psicológicos y sociales. Algunos estudios han problematizado el énfasis en estos enfoques, no solo porque no logran afectar realmente las conductas y los valores de las niñas, los niños y las y los adolescentes $[17,18]$, sino también porque amenazan sus derechos humanos fundamentales a la salud, la información y la vida $[19,20]$. Esto fue evidente en las conversaciones mantenidas con las niñas, los niños y las y los adolescentes, la mayoría de los cuales no había escuchado hablar nunca de DSR, pero sí de la sexualidad como algo malo que no deberían hacer.

Esto implica centrarse en las escuelas, responsables de la educación sexual de niñas, niños y adolescentes, pero también en los medios de comunicación y en los adultos que son referentes cercanos de estas personas. Como este estudio y otras investigaciones lo han mostrado [21], entre las niñas, los niños y las y los adolescentes menores de 14 años, las relaciones con sus madres, padres y cuidadores tienen mayor relevancia que las relaciones con sus pares. Esto supone grandes retos al momento de fortalecer estrategias entre pares, como las que suelen usarse para el trabajo de educación para la sexualidad y en SSR con niñas, niños y adolescentes. Además, se ha señalado que las intervenciones que involucran el fortalecimiento de la comunicación entre madres, padres o cuidadores, y sus hijas e hijos o menores a cargo, son más exitosas [22]. El trabajo con estas personas adultas puede contribuir al ejercicio de una parentalidad positiva, capaz de hacer frente a la influencia demostrada de la televisión en los comportamientos de las niñas, los niños y las y los adolescentes [21].

En el proceso de fortalecimiento de las niñas, los niños y las y los adolescentes como seres autónomos y cuidadosos de sí y de los otros, es también un imperativo superar las formas sexistas y heteronormativas de educación que se dan fuera y al interior de las aulas, y que hacen parte de su experiencia socializadora diaria [23]. A través de los mensajes que reciben, las niñas, los niños y las y los adolescentes construyen el significado de que "se es una mujer o un hombre de una vez y para siempre y de que la masculinidad y la feminidad son de- rivaciones 'naturales' de las características biológicas" [24, p. 2]. Esto inculca en las personas actitudes y valores negativos sobre la diversidad sexual y de género, $\mathrm{y}$ obstaculiza la posibilidad de construcciones diversas de sí, "en las que la masculinidad no esté asociada exclusivamente, con la violencia y el ejercicio del poder y la feminidad con la belleza física, la expresión emocional y las relaciones" [25, p. 36].

Las niñas son quizá las más afectadas con la falta de educación sexual integral, pues particularmente a ellas se dirigen mensajes de peligro y de control sobre los cuerpos con los que se culpabiliza la expresión de su sexualidad. Así, a las niñas se las responsabiliza del cuidado a las agresiones de las que son susceptibles víctimas, pero a los niños no se los educa para que no agredan. Otras investigaciones han identificado que "algunos niños suelen atribuir la responsabilidad de estos actos [de agresión sexual] a la mujer, por usar ropas atrevidas o provocadoras de los deseos masculinos incontrolables" y que "algunas niñas admiten esta responsabilidad" [16, p. 1020]. Estos mensajes y percepciones se han vinculado con la idea de que existen características intrínsecas al sexo/género, como la agresividad y la virilidad en los hombres e identidades masculinas, o la pasividad y la indefensión en las mujeres y las identidades femeninas [26].

Generalmente, se piensa que la vulnerabilidad de las niñas es una característica intrínseca a su sexo y a su edad. Sin embargo, ni el sexo ni la edad residen en las hormonas o en las psiques [27,28]. La edad y el tipo predominante de relaciones de género que impone roles y estatus en razón del sexo son construcciones sociales. Esto puede notarse en las variaciones existentes en las mismas categorías en diferentes lugares y tiempos, e incluso en la inexistencia de algunas categorías asociadas a la edad en el pasado, como la categoría "infancia" [28-30]. ¿Hasta qué punto, entonces, la vulnerabilidad de lo femenino - asociado por antonomasia a mujeres y niñas - no es una profecía que se cumple a sí misma?

Por supuesto, los discursos que limitan y desconocen los derechos de las personas tienen efectos en su salud. Mucha de la satisfacción que las niñas, los niños y las y los adolescentes mostraron con los servicios de salud que reciben se relaciona con el desconocimiento de sus derechos. Por eso, puede que se presenten barreras al acceso potencial de esta población a los servicios, entendidas estas como barreras del lado de la demanda, que afectan el deseo de buscar atención en salud [31]. Esto es más problemático, si se tiene en cuenta el contexto de pobreza en el que viven las y los entrevistados y el peso que esto tiene como determinante social de los resultados en salud $[8,9]$. 


\section{Agradecimientos}

Se agradece especialmente a las niñas, los niños y las y los adolescentes que participaron de las entrevistas, así como la lectura atenta y los comentarios al manuscrito realizados por Mariana Calderón Jaramillo, Sandra Marcela Sánchez y Juan Sebastián Arteaga.

\section{Fuente de financiación}

Esta investigación fue financiada por la Embajada de Canadá en Colombia y por la Asociación Profamilia.

\section{Conflicto de intereses}

Las personas en las que recae la autoría del texto declaran no tener ningún conflicto de interés.

\section{Declaración de responsabilidad}

Los puntos de vista expresados en este artículo son responsabilidad de sus autoras y autores, y no de la institución en la que trabajan o de la fuente de financiación.

\section{Declaración de autoría}

Todas las autoras y todos los autores participaron en la concepción y el diseño del artículo. Daniela Roldán realizó las entrevistas y dirigió los grupos focales, hizo el análisis preliminar de los datos y participó en la redacción del artículo. Andrea Arenas y Danny Rivera analizaron algunos datos y apoyaron la redacción del manuscrito. Juan Carlos Rivillas y Luz Janeth Forero revisaron críticamente el contenido y aprobaron la versión a publicar.

\section{Referencias}

1. Unicef. Derechos de los niños [internet]; s. f. [citado 2020 ene. 27]. Disponible en: https://www.unicef.org/colombia/derechosde-los-ninos

2. González M. Derechos sexuales: niños, niñas y adolescentes. En SELA (Seminario en Latinoamérica Teoría Constitucional y Política) Papers [internet]. 2009 [citado 2020 ene. 27];68:1-25. Disponible en: https://digitalcommons.law.yale.edu/yls_sela/68/

3. Colombia, Ministerio de Salud y Protección Social. Política nacional de sexualidad, derechos sexuales y derechos reproductivos. Bogotá: Ministerio de Salud y Protección Social; 2014.

4. Colombia, Congreso de la República. Ley 115, por la cual se expide la Ley General de Educación (1994 feb. 8).

5. Ministerio de Educación Nacional, Universidad de los Andes. Evaluación del Programa de Educación para la Sexualidad y Construcción de Ciudadanía (PESCC). Informe final. 2014.
6. Vargas Trujillo E. Sexualidad... Mucho más que sexo. Una guía para mantener una sexualidad saludable. Bogotá: Universidad de los Andes; 2007.

7. Organización Mundial de la Salud. Defining sexual health. Sexual and reproductive health. World Health Organization [internet]; 2006 [citado 2020 ene. 27]. Disponible en: http://www.who.int/ reproductivehealth/topics/sexual_health/sh_definitions/en/

8. Profamilia, Ministerio de Salud y Protección Social. Encuesta nacional de demografía y salud. Tomo II. Componente de salud sexual y salud reproductiva. Bogotá [internet]; 2017 [citado 2020 ene. 27]. Disponible en: https://profamilia.org.co/wp-content/ uploads/2019/05/ENDS-2015-TOMO-II.pdf

9. Murad R, Rivillas JC, Gómez G, et al. Determinantes del embarazo en adolescentes en Colombia. Explicando las causas de las causas. Bogotá: Profamilia; Fundación Plan [internet]; 2018 [citado 2020 ene. 27]. Disponible en: https://www.minsalud.gov. co/sites/rid/Lists/BibliotecaDigital/RIDE/VS/ED/PSP/informedeterminantes-sociales-embarazo-en-adolescente.pdf

10. Moore AM, Reynolds P. Childhood and Sexuality: Contemporary Issues and Debates. Basingstoke, UK: Palgrave MacMillan [internet]; 2017 [citado 2020 ene. 27]. Disponible en: https:// www.bookdepository.com/es/Childhood-Sexuality-Allison- MMoore/9781137524959

11. Profamilia. Proyectos sociales de alto impacto en salud sexual y reproductiva [internet]; s. f. [citado 2020 sep. 17]. Disponible en: https://profamilia.org.co/construyendo-comunidad/labor-social/

12. Departamento Administratio Nacional de Estadística (DANE). Colombia. Medición de pobreza monetaria y desigualdad 2018 [internet]; 2018 [citado 2020 ene. 27]. Disponible en: http://microdatos.dane.gov.co/index.php/catalog/608/get_microdata

13. Consejo de Organizaciones Internacionales de las Ciencias Médicas (CiOms), Organización Panamericana de la Salud, Organización Mundial de la Salud. Pautas éticas internacionales para la investigación relacionada con la salud con seres humanos. 4. ${ }^{a}$ ed. Ginebra: cIoms [internet]; 2016 [citado 2020 ene. 27]. Disponible en: https://cioms.ch/wp-content/uploads/2017/12/CIOMS-EthicalGuideline_SP_INTERIOR-FINAL.pdf

14. Cook RJ, Cusack S. Estereotipos de género. Perspectivas legales transnacionales. Pennsylvania: Pennsylvania Studies in Human Rights; 2009.

15. Pacheco-Sánchez CI, Rincón-Suárez LJ, Guevara EE, et al. Significaciones de la sexualidad y salud reproductiva en adolescentes de Bogotá. Salud Pública Mex. 2007;49(1):45-51.

16. Bolívar Bonilla C. Justificaciones morales de los niños y niñas acerca de la sexualidad. Rev Latinoam Ciencias Soc Niñez y Juv. 2010;8(2):1013-23.

17. Vivo S, López-Peña P, Saric D. Salud sexual y reproductiva para jóvenes. Revisión de evidencia para la prevención. Ciudad de México: Banco Interamericano de Desarrollo, División de Protección Social y Salud; 2012.

18. Malone P, Rodriguez M. Comprehensive sex education vs. abstinence-only-until-marriage programs. Hum Rights [internet]. 2011 [citado 2020 ene. 27]; Disponible en: https://www.americanbar. org/groups/crsj/publications/human_rights_magazine_home/human_rights_vol38_2011/human_rights_spring2011/comprehensive_sex_education_vs_abstinence_only_until_marriage_programs/

19. Santelli J, Ott MA, Lyon M, et al. Abstinence and abstinence-only education: A review of U.S. policies and programs. Journal of Adolescent Health. 2006;38:72-81. Dor: https://doi.org/10.1016/j. jadohealth.2005.10.006 
20. Ott MA, Santelli JS. Abstinence and abstinence-only education. Current Opinion in Obstetrics and Gynecology. 2007;19(5):44652. DOI: https://doi.org/10.1097/GCO.0b013e3282efdc0b

21. Vargas Trujillo E, Barrera F. Adolescencia, relaciones románticas y actividad sexual: una revisión. Rev Colomb Psicol. 2002;(11):115-34.

22. Finkelhor D, Dziuba-Leatherman J. Victimization prevention programs: A national survey of children's exposure and reactions. Child Abus Negl. 1995;19(2):129-39. DoI: https://doi. org/10.1016/0145-2134(94)00111-7

23. Renold E. "Coming out": Gender, (hetero)sexuality and the primary school. Gend Educ. 2000;12(3):309-26. Dor: https://doi. org $/ 10.1080 / 713668299$

24. Bilinkis M, García Palacios M. El desarrollo esperado: una aproximación a la sexualidad en la niñez desde las experiencias formativas en la escuela. Lúdicamente. 2015;4(7).

25. Vargas Trujillo E, Rojas Martínez ÁM, Balanta P. Televisión y género: un análisis desde la perspectiva de los niños y las niñas. Mediaciones. 2008;6(8):25-40. Dor: https://doi.org/10.26620/uniminuto.mediaciones.6.8.2008.25-40
26. Badinter E. Por mal camino. Madrid: Alianza; 2004.

27. Rubin G. Reflexionando sobre el sexo: notas para una teoría radical de la sexualidad. [internet]. 1989 [citado 2020 ene. 27]; 1-59. Disponible en: http://webs.uvigo.es/xenero/profesorado/beatriz suarez/rubin.pdf

28. Aries P. Capítulo II. El descubrimiento de la infancia. En El niño y la vida familiar en el Antiguo Régimen. [internet]; s. f. [citado 2020 ene. 27]. Disponible en: http://iin.oea.org/Cursos_a_distancia/El_nino_y_la_vida_familiar.pdf

29. Vila J. Historia de la infancia [reseña]. Educ Soc [internet]. 1982 [citado 2020 ene. 4]; (60):123-6. Disponible en: https://www.raco. cat/index.php/EducacioSocial/article/viewFile/296676/385608

30. López Y. De la inocencia del niño a la sexualidad infantil. Affect Soc [internet]. 1999 [citado 2020 ene. 27]; 2(4):1-15. Disponible en: https://www.researchgate.net/publication/47457370_De_la_ inocencia_del_nino_a_la_se xualidad_infantil

31. Restrepo-Zea JH, Silva-Maya C, et al. Acceso a servicios de salud: análisis de barreras y estrategias en el caso de Medellín, Colombia. Rev Gerenc Polit Salud. 2014;13(27):236-59. Dor: https:// doi.org/10.11144/Javeriana.rgyps13-27.assa 